\section{Point: Don't Call Me a DOC!}

(DD.M. Yousem

T he words we use matter. That is why we build lexicons. I want to petition for the removal of the phrase "disc osteophyte complex" (DOC) from the neuroradiology lexicon. I am seeing this phrase used very frequently in descriptions of every level of the cervical spine by residents coming to neuroradiology — and it galls me. To prepare for this editorial, I consulted the 3 most active orthopedic and neurosurgery spine surgeons at Johns Hopkins, and they endorsed my call to eliminate the use of disc osteophyte complex.

We can readily distinguish discs from osteophytes on the basis of our gradient-echo (GRE) low flip-angle thin-section cervical spine axial scans (Figure). On these sequences, the disc is bright and the osteophyte is dark. By contrast, on fast spin-echo T2weighted images both may be dark, so the inclusion of this gradient-echo scan is critical to accurate interpretation. Rarely, we may see a severely desiccated disc that defies this GRE classification, but for most cases, we can tell a disc (bright) from an osteophyte (dark), and they do not necessarily coexist. Why should we care? Because the spine surgeons do! They told me!

http://dx.doi.org/10.3174/ajnr.A4927
If the disease is exclusively a disc, the surgeons may attempt a discectomy alone, or they may do watchful waiting for the disc to regress. Jay Khanna, orthopedic surgeon, said, “The larger, more hydrated discs tend to resorb. So if the patient is neuro-intact, we can try to treat them nonoperatively." Osteophytes do not regress. If the disease is predominantly an osteophyte, it requires more drilling from an anterior approach, and according to Jean-Paul Wolinsky, neurosurgeon, it is a much more difficult procedure. He will warn the patient as such. Foraminotomies are more commonly required when osteophytes and uncovertebral joint spurs are present-the distraction of the intervertebral graft alone may not relieve the stenosis. From a posterior approach, Wolinsky says, a herniation is removed, whereas an "osteophyte is left alone, and instead the nerve is decompressed widely." If there is coexistent ossification of the posterior longitudinal ligament, the approach is exclusively posterior, with indirect decompression. Neurosurgeons Ali Bydon and Dan Sciubba state, "lumping DOC generically doesn't help us determine the best approach" and "differentiating the two can change the management."

Therefore, I implore you, my colleagues, to say that either there is a disc, there is an osteophyte, or there is a disc and osteophyte. Calling every level a disc osteophyte complex is a cop-out.

I used to say to my young children, "Use your words" (ie, do not hit). To my adult colleagues, I say, "Use your words wisely."

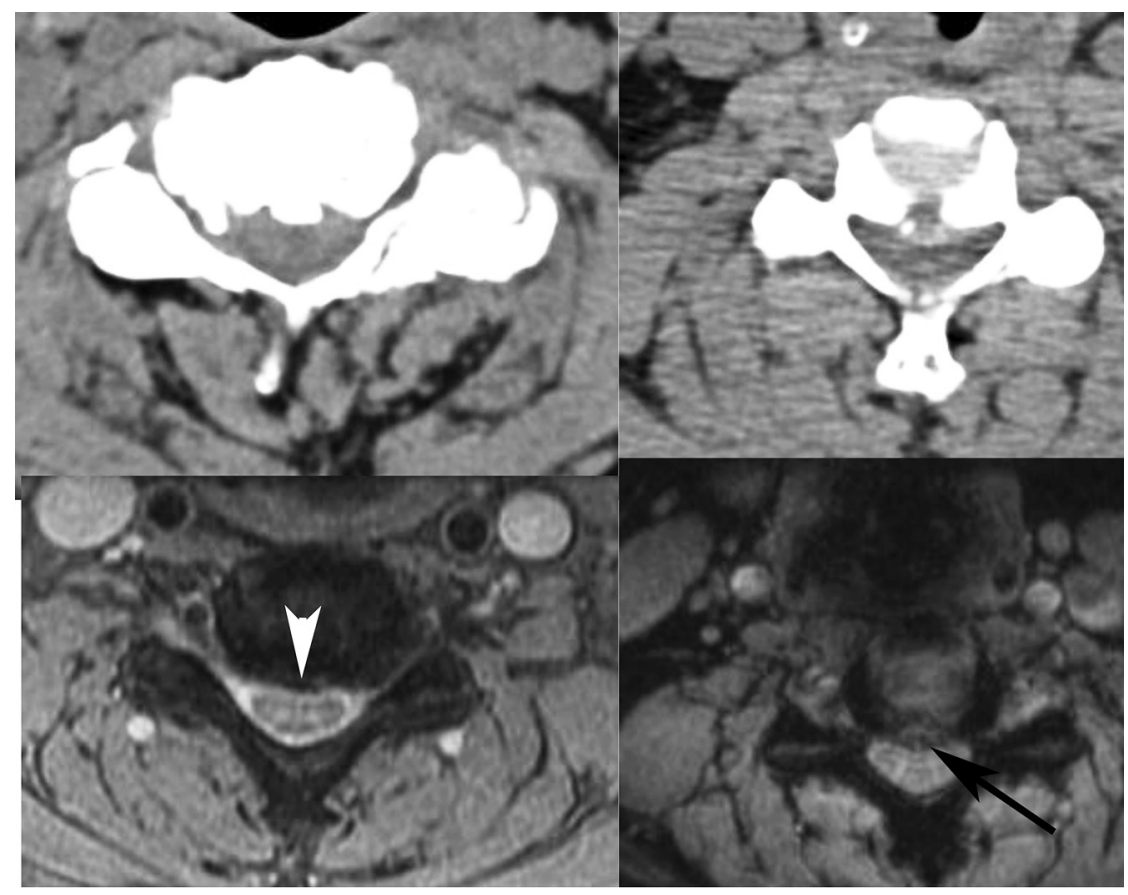

FIGURE. Disc bright, osteophyte dark. Note the high-intensity disc herniation (black arrow) to be distinguished from the low-intensity osteophyte (white arrowhead), with confirmatory corresponding CT scans above. 